\title{
Sociologie et sociolinguistique du franbreu
}

\author{
Ben-Rafael, Eliezer \& Ben-Rafael, Miriam \\ Université de Tel-Aviv et Chercheure indépendante \\ saba@post.tau.ac.il
}

\section{Introduction}

Cet article présente un ouvrage récent (Ben-Rafael / Ben-Rafael 2013) sur les francophonies israéliennes et suggère, à partir de là, de nouvelles voies d'investigations qui rejoignent les intérêts de chercheurs sociologues et sociolinguistes - dans la production langagière contemporaine.

Des enquêtes des années 1990 (Ambassade 1995) ont montré que plus de 20\% des adultes israéliens ont quelques connaissances du français. Parmi cette population, 250000 à 300000 personnes pourraient être considérées comme «francophones». Comme nous l'avons montré dans notre livre, Israël fait l'expérience simultanée de plusieurs francophonies originales qui diffèrent par leurs sources aussi bien que leurs expressions. Un premier modèle est le français vernaculaire (voir Boudras-Chapon 2008) des immigrants de classes défavorisées originaires d'Afrique du nord. Un second modèle concerne les immigrants de même origine ainsi que des immigrants francophones d'autres pays où le français était en son temps considéré comme la langue de culture par excellence (surtout les pays balkaniques, la Turquie ou l'Egypte). Un troisième modèle plus récent date de cette vague d'immigration de France qui débuta dans les années 1990 dans le contexte du malaise de la population juive face à la judéophobie croissante qui sévit durant ces années sur le continent européen.

Tous ces modèles se trouvent en Israël en contact direct et permanent avec la langue légitime, l'hébreu. Sous cette influence, le français évolue chez les francophones de toute origine vers une forme hybride que M. Ben-Rafael (2011) avait déjà dénommé le «franbreu » (voir le cas parallèle de l'anglais israélien, Hebrish, chez Olshtain, Blum-Kulka 1989). Même si la vague récente d'immigration francophone manifeste avec plus de détermination que toute autre le désir de maintenir 'son' français tout en 's'hébraïsant', il est inéluctable que cette langue converge vers le franbreu dans un avenir plus ou moins proche. C'est dans ce contexte que le travail présenté ici se focalise sur le parcours sociologique et l'évolution sociolinguistique du franbreu et considère certains prolongements qu'il laisse entrevoir.

Comme nous l'avons mentionné, durant les années 1950 et 1960, un grand nombre de personnes en provenance d'Afrique du Nord ont immigré en Israël (E. Ben-Rafael, Sharot 1991). Pour ceux parmi ces immigrants qui avaient reçu une éducation secondaire ou supérieure, et avaient joui d'un statut social moyen dans un environnement urbain, le français était la langue quotidienne et de culture, même si subsistaient dans sa pratique des accents caractéristiques et certains éléments judéo-arabes et arabes. Ceux, en revanche, qui n'avaient reçu qu'une éducation primaire et étaient issus de couches sociales populaires, parlaient le plus souvent un français populaire conjointement au judéo-arabe.

Ces immigrants qui ne faisaient pas partie des classes moyennes au départ se sont généralement regroupés en communautés dont le niveau socioéconomique les apparentait aux couches défavorisées de la société (Smooha 1972). Comme d'autres groupes - yéménites, kurdes ou libyens - qui ont connu des expériences analogues, ces Nord-Africains ont eu tendance à reproduire dans un esprit traditionnel, des modèles de vie coutumiers : fréquentation de synagogues ethniques, pèlerinages sur les tombes d'hommes vénérés, festivals et rituels de mariage. Malgré cet attachement au passé, l'environnement israélien devait altérer les comportements à travers l'influence du système scolaire, le service militaire, les nouvelles occupations et les contraintes d'une société de consommation. 
Le facteur principal de la mutation a été l'acquisition de l'hébreu et sa pratique, le passage à l'hébreu moderne étant légitimé par le fait qu'il s'agissait de la langue de la Bible commune à tous les héritages judaïques. En quelques années à peine, toute cette nouvelle population contrôlait grosso modo la langue légitime, ce qui, cependant, n'impliquait pas l'abandon total et immédiat du judéo-arabe ou du français populaire. Plusieurs au sein de la deuxième génération ont hérité ces codes de leurs parents, tout au moins en partie (Hofman, Fisherman 1972). Cependant, le français a difficilement survécu parmi les descendants ultérieurs des Juifs nord-africains dans les strates sociales défavorisées. Le sort du français fut différent surtout chez les immigrants de même origine qui arrivèrent en Israël avec des ressources culturelles et matérielles et accédèrent à la classe moyenne.

Ces immigrants-là avaient été immergés dans la culture française durant des décennies (Chouraqui 1965). En Israël, ils ont appris l'hébreu et se sont facilement intégrés, mais le français est resté, à leurs yeux, un marqueur essentiel de distinction qu'ils se sont efforcés de préserver. Ils se sont confondus avec ces immigrants, moins nombreux, de France ou d'autres pays francophones, pour qui le français était également la première langue. Ces contingents se joignirent également, de ce point de vue, aux arrivants des Balkans et du Moyen-Orient qui avaient adopté le français comme seconde langue à une époque où il jouissait un peu partout du prestige de langue culturelle par excellence. Notons ici que dans des pays comme l'Égypte, la Turquie ou le Liban, le français était même devenu très souvent une langue première au même titre que la langue légitime locale.

En Israël, dans les années 1950-1970, l'idéologie dominante exigeait impérativement l'adoption de l'hébreu. Tous les immigrants, francophones et autres, étaient donc tenus d'acquérir et de pratiquer l'hébreu pour s'intégrer rapidement dans leur nouvelle nation (Myhill 2006; M. Ben-Rafael 2001a). Ces pressions toutefois n'ont pas empêché les francophones, comme les autres locuteurs, d'utiliser leurs codes d'origine, ne fût-ce qu'entre conjoints et amis, ou encore dans leurs lectures ou autres activités culturelles ${ }^{0}$.

\section{Un français transnational}

La situation changea du tout au tout avec la vague d'immigration francophone qui débuta vers la fin des années 1980 avec 2000 à 5000 immigrants par an. En 2011, on estime que près de 50000 personnes vivant en Israël sont issues de cette immigration. Ces nouveaux venus (NVs) constituent un type de population, inconnu jusqu'alors, d'immigration francophone. Son parcours singulier se manifeste d'abord dans le paysage linguistique des lieux où ses membres ont tendance à s'installer (E. Ben-Rafael, M. BenRafael 2008). À Natanya, par exemple, où ils sont nombreux, le français est prédominant sur les enseignes et les panneaux, depuis les agences immobilières, les cafés, les restaurants, les boutiques, et jusqu'aux synagogues. Le français y est omniprésent, parfois de manière exclusive, parfois à côté de l'hébreu, de l'anglais et du russe. La multiculturalisation que connaît la société israélienne depuis les années 1980, avec l'émergence de forces politiques locales ethniques ou ethno-religieuses explique la facilité des nouveaux immigrants à afficher leur particularisme en public. Ce changement de climat convient aussi pour le mieux à ces immigrants qui ont tendance à vouloir illustrer le modèle de diaspora transnationale à l'heure où il se multiplie à travers le monde dans le contexte de la mondialisation (voir Glick Schiller et al. 1992). Comme beaucoup d'autres cohortes d'immigrants actuels du même type, ces NVs aspirent à s'insérer dans leur nouvel environnement tout en maintenant une forte solidarité et des liens institutionnels avec leur société d'origine.

Tableau 1 : NVs vs Vatikim : caractéristiques, attitudes envers Israël, contacts avec la France

\begin{tabular}{|l|l|l|}
\hline a. Caractéristiques générales & Vatikim (643; \%) & NVs (215; \%) \\
\hline Éducation supérieure & 38 & 59 \\
\hline Religieux & 18 & 51 \\
\hline Séculier & 44 & 12 \\
\hline
\end{tabular}




\begin{tabular}{|c|c|c|}
\hline Âgé/e de moins de 40 ans & 32 & 48 \\
\hline Origine nord-africaine (sujet ou parent) & 61 & 74 \\
\hline \multicolumn{3}{|c|}{ b. La qualité de vie en Israël en comparaison avec la France $(659 ; \%)\left(\gamma^{2}=0.00\right)$} \\
\hline Meilleure en Israël & 7.2 & 52.2 \\
\hline La même chose & 9.1 & 19.7 \\
\hline Meilleure en France & 13.7 & 28.1 \\
\hline Total & 100.0 & 100.0 \\
\hline \multicolumn{3}{|c|}{ c. Contacts avec des amis en France $(840 ; \%)\left(\gamma^{2}=0.00\right)$} \\
\hline Quotidiens & 3.8 & 2.7 \\
\hline Hebdomadaires & 21.5 & 11.9 \\
\hline De temps à autres & 56.5 & 36.1 \\
\hline Pas de contact & 18.2 & 49.3 \\
\hline Total & 100.0 & 100.0 \\
\hline
\end{tabular}

Une recherche sur les citoyens français d'Israël confirme que les NVs arrivés dans le pays depuis 1985 et les Vatikim (vétérans) arrivés précédemment (E. Ben-Rafael, Sternberg 2009) sont sensiblement différents les uns des autres (tableaux 1 et 2).

Le tableau 1 montre que les NVs sont relativement plus nombreux que les Vatikim à avoir reçu une éducation supérieure, ce qui signifie aussi qu'ils ont de meilleures chances d'atteindre des strates professionnelles élevées. Les NVs sont également plus jeunes, ce qui présuppose une entité socialement plus ambitieuse. Par ailleurs, les nouveaux venus sont plus religieux et plus traditionnalistes que les Vatikim, même si la plupart sont originaires du même pays, l'Afrique du nord ${ }^{1}$. Parallèlement, les nouveaux venus semblent plus satisfaits des conditions de vie rencontrées en Israël que les Vatikim, tandis qu'ils font état de relations plus intenses et plus régulières avec la France, ce que souligne le tableau 2.

Tableau 2 : Comportement linguistique des Israéliens de nationalité française

\begin{tabular}{|l|l|l|l|}
\hline & NVs & Vatikim \\
\hline & $\begin{array}{l}\text { Arrivé depuis 20 ans ou } \\
\text { moins }\end{array}$ & Arrivé il y a 20 ans ou plus & $\begin{array}{l}\text { Né en Israël ou } \\
\text { arrivé avant l'âge } \\
\text { de 8 ans }\end{array}$ \\
\hline a. Langue parlée avec le partenaire $(718 ; \%)$ & 36.1 & 5.4 \\
\hline Français & 71.3 & 24.1 & 12.5 \\
\hline Fr et H & 9.8 & 36.9 & 81.1 \\
\hline Hébreu & 15.5 & 2.9 & 1.0 \\
\hline Autre & 3.4 & 100.0 & 100.0 \\
\hline Total & 100.0 & 19.1 & 4.4 \\
\hline b. Langue parlée avec ses enfants $(640 ; \%)\left(\gamma^{2}=0.00\right)$ & 28.1 & 15.0 \\
\hline Français & 67.7 & 51.6 & 79.7 \\
\hline Fr et Hébreu & 22.2 & 1.2 & 0.9 \\
\hline Hébreu & 8.9 & 100.0 & 100.0 \\
\hline Autre & 1.2 & \\
\hline Total & 100.0 & 23.6 & 1.9 \\
\hline c. Langue principale utilisée pour la lecture $(846 ; \%)\left(\gamma^{2}=0.00\right)$ & \\
\hline Français & 50.0 &
\end{tabular}




\begin{tabular}{|l|l|l|l|}
\hline Fr et Hébreu & 33.0 & 52.8 & 14.7 \\
\hline Hébreu & 9.9 & 18.5 & 74.9 \\
\hline Autre & 7.1 & 5.1 & 8.5 \\
\hline Total & 100.0 & 100.0 & 100.0 \\
\hline d. Langue principale utilisée pour regarder des programmes de TV $(838 ; \%)\left(\gamma^{2}=0.00\right)$ & \\
\hline Français & 19.5 & 13.0 & 2.8 \\
\hline Fr et Hébreu & 46.3 & 56.3 & 33.6 \\
\hline Hébreu & 19.5 & 20.4 & 45.7 \\
\hline Autre & 14.7 & 10.3 & 17.9 \\
\hline Total & 100.0 & 100.0 & 100.0 \\
\hline e. Langue principale utilisée par les enfants entre eux $(404 ; \%)\left(\gamma^{2}=0.00\right)$ & \\
\hline \multicolumn{7}{|l|}{ Parents en Is : moins de 20 ans } & Parents en Is : 20 ans \\
\hline Français & 23.5 & 6.0 \\
\hline Fr et Hébreu & 27.2 & 11.2 \\
\hline Hébreu & 42.6 & 80.6 \\
\hline Autre & 6.7 & 2.2 \\
\hline Total & 100.0 & 100.0 \\
\hline
\end{tabular}

Les NVs diffèrent également des Vatikim par leurs comportements et leurs attitudes linguistiques : leur pratique culturelle et leurs activités sont marquées par une forte allégeance à la langue et la culture françaises. La plupart d'entre eux conservent le français comme la langue des échanges au sein de la famille et dans la vie sociale - ce qui n'est pas caractéristique des Vatikim. Le français cède la place à l'hébreu comme la langue la plus utilisée par les enfants entre eux, cette tendance étant toutefois moins évidente chez les NVs que chez les Vatikim.

Ces traits sont caractéristiques de la diaspora transnationale des nouveaux venus qui s'insèrent dans la société tout en souhaitant se regrouper dans des espaces qu'ils ont choisis et en maintenant des relations avec leur pays et leur communauté d'origine. Ces nouveaux venus manifestent leur volonté d'apprendre l'hébreu et la culture hébraïque sans renoncer au français. Par ailleurs, leurs études et leurs professions ne peuvent les rendre socialement assimilables aux couches défavorisées dans lesquelles on retrouve de nombreuses personnes d'origine nord-africaine des vagues d'immigration précédentes.

Les nouveaux venus contrastent également avec leur environnement social direct à plusieurs égards. Ils ont tendance à être religieux contrairement à la plupart des Israéliens de classe moyenne. Le français qui est leur première langue les différencie de ces Israéliens hébréophones pour qui la langue seconde est le plus souvent l'anglais. Leur orientation culturelle en direction de la France contraste aussi avec l'intérêt pour les États-Unis, caractéristique des autres milieux de cette classe moyenne. Les nouveaux venus constituent donc en Israël un nouvel élément social aussi bien qu'un type original de population francophone.

On peut s'attendre à ce que le français de ces nouveaux venus reflète, avec le temps, l'influence du contact avec l'hébreu, et qu'il se rapproche du franbreu des anciens immigrants francophones. Mais d'autre part, il y a également le fait que cette population produit des cadres d'activités et de socialisation dans lesquels le français - nous entendons le français normatif - reste prédominant. Ces cadres structurent la dimension communautaire de cette population et concrétisent son caractère transnational.

Sous l'impulsion des NVs, mais à l'intention des francophones israéliens en général, depuis les années 1990, associations et sites internet se sont multipliés en direction des francophones d'Israël aussi bien que des communautés juives de France et des pays de langue française en général. Ces initiatives sont surtout dues aux nouveaux venus et aux milieux juifs en France. Les associations de langue française étaient, 
jusqu'à l'arrivée des nouveaux venus, pratiquement inexistantes en Israël, si ce n'est une douzaine de cercles rassemblant quelques centaines de membres au total. Aujourd'hui, la situation a radicalement changé. Plus de quatre-vingts associations d'ampleur diverse se répartissent en fonction de leurs domaines d'activité et de leurs objectifs.

Dans l'ensemble, ces associations forment un tableau assez cohérent d'intérêts communautaires. Certaines mettent l'accent sur les préoccupations sociales et humanitaires et ciblent la population israélienne en général; elles cherchent à exprimer de façon tangible la contribution de la présence francophone à la construction de cette société. Un autre groupe d'associations traite des problèmes que les immigrants francophones rencontrent dans leur insertion sociale. Certaines sont indépendantes et locales ; d'autres sont des branches franco-israéliennes d'institutions juives internationales de bienfaisance (WIZO ou le Bnei Brith); d'autres encore travaillent en coordination avec des antennes en France. Elles mobilisent des compétences, notamment dans le marché de l'emploi, le logement, ou la gestion financière. Une autre catégorie d'associations vise les immigrants de groupes professionnels spécifiques : hommes ou femmes d'affaires, médecins, avocats ou étudiants. Ces associations et leurs activités dépendent de leur public. Un groupe supplémentaire se consacre à des activités culturelles en langue française : conférences, débats, concerts ou expositions; elles ont pour but de multiplier le nombre de foyers d'activités culturelles et sociales de langue française.

Un autre élément participe à construire une communauté : c'est le réseau qui, depuis les années 1990, ne cesse de croître, constitué par les sites internet francophones se disputant l'attention des personnes intéressées - des nouveaux venus la plupart du temps. Certains sont très actifs, régulièrement mis à jour, et visités par des milliers de surfeurs ; d'autres sont éphémères et ne touchent qu'un public restreint. Deux catégories de sites sont plus importantes: les sites informatifs, et ceux dédiés à des sujets culturels, politiques, ethniques ou religieux. Certains coopèrent avec des équipes basées en France, constituant des initiatives binationales. Ils accordent une attention spéciale aux sujets qui relient les francophones d'Israël aux Juifs de France et d'autres pays francophones.

\section{Le franbreu}

Cette expérience de vie qui se veut communautaire et qui marque un bon nombre de NVs tend, à divers degrés, à s'étendre dans la population francophone. Plus qu'auparavant, les anciens francophones sont réceptifs aux activités associatives ou culturelles s'adressant à eux et on les voit plus nombreux aux manifestations organisées ici et là par des acteurs sociaux engagés. Ce sont là des opportunités nouvelles de pratiquer le français et d'y intéresser les enfants. Ce français, cependant, n'est pas sans subir, même chez les NVs les plus attachés à l'utilisation de la langue française, l'influence de l'apprentissage de l'hébreu et de son utilisation généralisée dans la vie publique israélienne (voir M. Ben-Rafael, Schmid 2007).

$\mathrm{Au}$ cours des années, la maîtrise du français est marquée par la présence de l'hébreu dans l'environnement, son apprentissage par les locuteurs et les conditions dans lesquelles ils évoluent. Les francophones peuvent partager un sentiment de perte linguistique et rencontrer des difficultés à s'exprimer et retrouver les mots appropriés pour traduire leurs idées. Ils sont encore et toujours persuadés qu'il est impératif de parler l'hébreu pour maintenir leur identité israélienne, mais, souvent ils n'attachent pas moins d'importance à concilier leur aspiration à devenir pleinement hébréophones tout en préservant le capital linguistique que représente la maîtrise du français.

Volens nolens, il est indiscutable que leur français se modifie sous l'influence de l'hébreu dans leur activité langagière. Ce sont ces influences et leurs empreintes sur le développement du français qui ont engendré le franbreu. Le franbreu est un code dynamique qui est le produit du contact français-hébreu dans la vie courante. Cette variété orale ne porte pas atteinte à la qualité de l'expression des locuteurs en français standard dans le domaine culturel ${ }^{2}$. Dans la pratique orale, le franbreu s'exprime dans le lexique, la syntaxe et la morphologie. Les recherches sur les contacts de langue, en général, ont révélé une variété de modèles (Grosjean 1982 ; Romaine 1989 ; Gardner-Chloros 2009). La littérature parle d'interférences, de calques, d'emprunts lexicaux, de code switching, de simplification, d'attrition, et d'innovations 
grammaticales et lexicales. Ces modèles caractérisent les langues qui, de majoritaires deviennent minoritaires dans de nouvelles circonstances (Jacobson 1998; 2001 ; Bentahila1983 ; Meyers Scotton 1991). C'est dans cette optique que M. Ben-Rafael (2001a, 2001b) a examiné le français d'anciens immigrants francophones de classe moyenne $(n=150)$. Elle a constaté que le franbreu est une bonne illustration des tendances universelles relevées dans les situations de contacts de langues en général, ainsi que des particularités de la langue française dans de telles situations.

Le franbreu n'est qu'un exemple, parmi d'autres, d'hybridation linguistique relevé en Israël. La recherche a montré l'influence de l'hébreu sur l'anglais (Olshtain, Blum-Kulka 1989), l'espagnol (Berk-Seligson 1986), l'arabe (Koplewitz 1990) et l'allemand (Fishman, Kressel 1974), ainsi que l'influence de nombreuses langues sur le français en situation de contact (Pergnier éd. 1988) - par exemple, l'anglais au Québec ou à Welland (Mougeon et Beniak 1989), le flamand en Belgique (Witte, Beatens Beardsmore éds. 1987), l'arabe au Maroc (Bentahila 1983) ou l'alsacien en Alsace (Tabouret-Keller 1985).

Les recherches sur le franbreu de $\mathrm{M}$. Ben-Rafael ont mis à jour une répétition de phénomènes connus dans les études de contact de langues. On remarque ainsi la fréquence élevée d'occurrences de code switching (CS) entre français et hébreu qui vont de l'insertion en français d'une unité en hébreu ou emprunt (CSU), à celle de longs segments en hébreu (CSS). Comme le montre la bibliographie (Pergnier, éd. 1988), les noms constituent la grande majorité des emprunts, suivis par les phatiques et les adverbes ; les adjectifs, les conjonctions et les verbes sont moins nombreux. Les emprunts communautaires sont plus fréquents que ceux qui répondent à des préférences individuelles. En outre, la plupart des emprunts sont des substituts à des termes équivalents en français, ou bien viennent combler l'absence de termes appropriés dans cette langue.

Certains CSU peuvent alterner avec leurs équivalents français ; d'autres, en revanche, n'alternent jamais, au risque sinon de perdre leur signification exacte dans la traduction littérale. Les termes «mariage » et « khatuna » (mariage), par exemple, peuvent être utilisés en alternance, mais « khupa (la cérémonie de mariage) est spécifique à l'hébreu. On utilise indifféremment «memchala» ou gouvernement, mais seulement « kneset» (parlement). En règle générale, les CSU-noms concernent surtout la sphère publique (voir Tableau 3).

Les emprunts structurent aussi le discours de sujets spécifiques tels que la religion, l'immigration ou la politique. Même lorsque l'emprunt est traduisible en français, le locuteur préfère employer des termes en hébreu ; il choisira "morim» pour désigner les instituteurs dans le domaine de l'éducation, «misrad habriut » à propos du ministère de la Santé, « nituakh » pour opération chirurgicale.

Les instances de code switching ont des rôles très variés dans la conversation. Elles viennent, par exemple, exprimer les sentiments personnels; ce sont souvent des marqueurs subjectifs et identitaires (voir tableau 3). Elles facilitent des développements discursifs et des juxtapositions rhétoriques; elles marquent des tournants dans le discours et signalent des rapports de discours indirect. Des cas de code switchings apparaissent également dans des structures formelles, des expressions idiomatiques, ou des formules de félicitations. Dans l'ensemble, ces passages en franbreu d'une langue à l'autre se font naturellement, et les instances de code switching (CSU et CSS) n'entravent pas la fluidité de la parole.

Tableau 3 : Exemple de CS se rapportant à différents contextes

\begin{tabular}{|l|l|l|l|}
\hline \multicolumn{2}{|l|}{ CSU : au kibbutz } & \multicolumn{2}{l|}{ CSU : institutions publiques et fonctions } \\
\hline khaverim & membres du kibbutz & khaver kneset & membre du parlement \\
asefot & assemblées & avoda & parti socialiste \\
vaadot & comités & kupat kholim & mutuelle santé \\
markolit & épicerie & bituakh machlim & assurance santé \\
chkhuna & quartier & machkanta & hypothèque \\
\hline
\end{tabular}




\begin{tabular}{|c|c|c|c|}
\hline $\begin{array}{l}\text { arvut adadit } \\
\text { garin } \\
\text { chaliakh } \\
\text { tnua }\end{array}$ & $\begin{array}{l}\text { aide communautaire } \\
\text { groupe de pionniers } \\
\text { émissaire } \\
\text { mouvement de jeunes }\end{array}$ & $\begin{array}{l}\text { arnona } \\
\text { bituakh léumi } \\
\text { pardes } \\
\text { falkha }\end{array}$ & $\begin{array}{l}\text { impôt local } \\
\text { sécurité sociale } \\
\text { orangeraie } \\
\text { espace agricole }\end{array}$ \\
\hline \multicolumn{2}{|l|}{ Expressions d'émotions } & mifalim & usines \\
\hline tsarot & soucis & pkida & secrétaire \\
\hline mesubakh & compliqué & roe khechbon & expert comptable \\
\hline jafe meod & très bien & ganenet & jardinière d'enfants \\
\hline chalem im atsmi & je me sens bien & ozeret bajit & femme de ménage \\
\hline guch zar & étranger & tafkid & rôle \\
\hline le-mazalenu & pour notre chance & akhrai & responsable \\
\hline $\begin{array}{l}\text { ze ma jech } \\
\text { al tagidi jije be-seder, } \\
\text { af paam lo be-seder }\end{array}$ & $\begin{array}{l}\text { c'est ce qu'il y a } \\
\text { ne dis pas tout ira bien } \\
\text { ce n'est jamais bien }\end{array}$ & atsmai & indépendant \\
\hline
\end{tabular}

Concernant le lexique, le franbreu comporte des déviations du français standard. Les interférences lexicales et les calques sont nombreux (voir exemple 1, tableau 4), tandis qu'on constate une tendance à la réduction sémantique des termes français, véritables calques de leurs équivalents hébreux (exemples 2 et 3, tableau 4). Bien des phatiques sont également des calques; les confusions lexicales ne sont pas rares ; des mots passe-partout comme « truc », « machin » ou « chose » se substituent aux termes français problématiques. Le verbe « faire » est particulièrement populaire et prend la place de termes plus précis mais oubliés par le locuteur; les répétitions lexicales sont abondantes. Parallèlement, on note une tendance pour les innovations lexicales; de nouveaux verbes apparaissent, qui combinent des radicaux hébreux et des suffixes français (avec une priorité accordée au premier groupe verbal français) (voir exemples 4 et 5, tableau 4).

La grammaire franbreu, pour sa part, multiplie les écarts syntaxiques par rapport au français. Sous l'influence du contact français/hébreu, par exemple, le système verbal tend à se simplifier : le futur prend la place du conditionnel; le subjonctif est souvent absent. Ces tendances sont le reflet de l'influence de l'hébreu où ces deux modes sont inexistants (exemples 6 et 7, tableau 4).

De surcroît, en franbreu, les valences verbales sont très souvent différentes de celles du français standard, remplacées par des calques d'équivalents en hébreu (exemple 8, tableau 4). Une source supplémentaire de confusion vient de la non-correspondance des pronoms relatifs : «que » prend la place de «qui », «dont» et « où », comme en hébreu où l'on ne trouve que la forme « che » (exemple 9, tableau 4). De plus, les pronoms français «y» et «en», sans équivalents hébreux, sont rares en franbreu; «y» est éventuellement remplacé par des adverbes français, comme « là » ou « là-bas » (exemple 10, tableau 4).

Tableau 4 : Exemples choisis de déviations lexicales et grammaticales du franbreu par rapport au français standard

(1) j'ai fermé $28 \mathrm{~kg}$ / comme en hébreu : sagarti esrim ve-shmona kilo - pour : j’ai perdu $28 \mathrm{~kg}$

(2) chanson prend le sens de : poème - comme en hébreu le terme : chir

(3) métier (profession) devient aussi : matière scolaire/comme en hébreu le terme : miktsoa 
(4) tsilumer (de $\mathrm{H}$ tsilum/photo +er, suffixe du premier groupe verbal français) à la place du terme français : photographier

(5) fisfuser (de : fisfus/échec+ er), à la place de : échouer

(6) si ils seront au tikhon, si je vivrai je leur dirai d'apprendre le français

Au lieu de : s'ils vont au lycée, si je vis, je leur dirai d'apprendre le français

(7) elle préfère que je vais en Italie - au lieu de : elle préfère que j'aille en Italie

(8) elle aide à sa mère, au lieu de : elle aide sa mère

(9) tu me donnes ce que j'ai besoin - au lieu de : tu me donnes ce dont j'ai besoin

(10) je vais souvent là - au lieu de : j'y vais souvent

Sous l'influence de l'hébreu, le franbreu tend donc à se différencier de la norme française par les instances de code switching, les interférences, la tendance à la simplification et les innovations. Ces développements attestent d'une certaine nostalgie du français, mais dans le même temps, ils reflètent l'expérience de vie des locuteurs. Les instances de code switching par exemple, qui sont le trait majeur du franbreu, ne résultent pas simplement de l'oubli du français; ils sont aussi de nouveaux outils à la disposition des locuteurs dans la construction du discours.

En résumé, d'une part la recherche montre que le français des francophones de classe moyenne se transforme, notamment la langue orale, au contact de l'hébreu. D'autre part, les franbréophones estiment que le prix qu'ils ont payé pour former une génération de continuateurs hébréophones n'a pas été entièrement justifié. En faisant le choix de ne pas parler français avec leurs enfants mais seulement entre eux, la plupart ont renoncé à garantir le transfert de la langue tandis que leur langue évoluait vers le franbreu.

\section{Les prolongements du franbreu : un nouvel agenda}

Dans le contexte de la révolution actuelle de la communication, langue orale à son point de départ, le franbreu se caractérise aussi de plus en plus par des utilisations écrites. Si aujourd'hui la communication orale a pris une nouvelle importance grâce aux nouveaux moyens d'interaction électronique, ce même développement a amplifié la production écrite. Ordinateur, téléphonie électronique, communication internaute, tablettes et autres ont donné à l'écriture un essor sans précédent. On passe dorénavant énormément de temps à l'écriture - pour soi-même (notes, listes, réflexions), comme à l'intention d'autrui (sms, mails ou autres formes de messages). Ces formes d'écriture créent des codes singuliers dénommés dans un sens large 'langage sms'. Ces sociolectes écrits d'un type nouveau engendrent des normes orthographiques, lexicales ou grammaticales en marge de la langue normative : réduction de la longueur de l'énoncé, échanges rapides laconiques, transcriptions d'effets phonétiques.

Le franbreu lui aussi s'inscrit dans cette nouvelle profusion de productions écrites. Des formes typiques $\mathrm{du}$ franbreu oral deviennent très naturellement des composantes des langages sms et mail. On note surtout une profusion d'emprunts hébreux écrits en caractères hébreux ou latins : chalom, beseder ou nechikot à la place de salut, ça va ou bisous sont des exemples qui reviennent fréquemment dans les textes écrits. Des alternances de codes, unitaires ou segmentaires se retrouvent dans les textes écrits franbréophones des plus simples aux plus complexes.

Dans la rubrique mémo des téléphones portables, par exemple, on retrouve des listes d'épiceries qui comprennent aussi bien des items inscrits en français ou en hébreu, ou les deux à la fois; les termes hébreux apparaissent en caractères hébreux ou latins. On peut ainsi trouver, l'un à la suite de l'autre, des items tels que : Mont blanc suisse (fromage à tartiner), Milki (yaourt), carottes, ariel nozli (savon liquide), 
klementinot (clémentines), lekhem parus (pain coupé), fraises, bananot (bananes), pilpelim (poivrons), boissons, pasta (pâtes), chemen (huile), tsnoniot (petits radis).

Ce franbreu écrit cependant s'exprime le plus dans les échanges, qu'il s'agisse de franbréophones entre eux ou de franbréophones avec des proches hébréophones qui ont une certaine familiarité avec le français. Il faut noter ici également l'effet de l'atmosphère multiculturelle qui règne aujourd'hui dans le pays et qui est beaucoup plus tolérante envers la diversité linguistique que par le passé. C'est, à présent, l'occasion ultime pour les parents ou grands-parents fanbréophones qui pendant longtemps se sont refusés à parler français avec leurs enfants par identification idéologique, de transmettre quelques notions de français à leurs petits-enfants ainsi qu'aux parents de ces derniers, grâce à ces échanges électroniques. Ainsi, dans les sms actuels, on retrouve souvent des conversations écrites où alternent le franbreu écrit du locuteur franbréophone et l'hébreu écrit des locuteurs hébréophones :

Exemple 1. De retour en Israël après un long voyage, M. envoie un mail à son amie franbréophone comme elle :

Lajla tov (bonne nuit) Rivkele ! Nous voici de retour [---] toutes sortes de problèmes techniques instalatsia (plomberie). Tov (bon) tu dois être en pleine activité khevratique (sociale) avec ta famille de khutz laarets (l'étranger)

Exemple 2. E. (parent franbréophone) et sa fille L. (hébréophone) échangent un sms :

E. ton fils va mieux? Il va au gan (jardin d'enfants) aujourd'hui? Ma tchuvatekh (réponds-moi). Et toi comment ça va?

L. ha rega samti oto ba gan (je viens de l'amener au jardin d'enfants)

Dans ces messages franbréophones adressés à des hébréophones maîtrisant partiellement le français, les alternances de codes (en particulier les longues séquences) sont fréquentes; on retrouve souvent des répétitions voulues ou des passages écrits en français avec leur traduction en hébreu pour assurer la compréhension. De plus, lorsque l'interlocuteur franbréophone ressent une émotion particulière, il s'exprimera généralement en hébreu. Dans l'exemple suivant, où $S$. (hébréophone) et sa mère $M$. (franbréophone) communiquent par sms, la mère s'inquiète à propos de sa petite-fille. Après une première séquence en français, elle continue en hébreu (en caractères hébreux) sans hésitation jusqu'à la fin de leur échange :

Exemple 3.

M. j'espère que Liane se sent mieux. Bonne nuit.

S. lo olekh tov letsaari. Liane mamach lo beseder... mekava chemakhar jije akheret (ça va pas bien malheureusement. Liane se sent vraiment mal... espère que demain ça sera autrement)

M. ma amar harofe bedijuk? Ma h u natan la? (qu'est-ce-que le docteur a dit exactement? qu'est-ce-qu'il lui a donné)

S. moksipen ... khochev ze virus (moksipen...pense c'est un virus)

M. lo hevanti... khochev o lo khochev che ze virus? (pas compris... pense ou ne pense pas que c'est un virus)

Ces échanges caractérisent encore les contacts entre franbréophones israéliens et francophones vivant hors d'Israel qui maîtrisent partiellement l'hébreu. Une correspondance transnationale franbreu se met en place :

Exemple 4. Des amis de France envoient un mail à un couple franbréophone à l'occasion de la nouvelle année :

\footnotetext{
- Nous vous souhaitons chana tova ve metuka ve bria ve chalom (une bonne année et douce et saine et la paix)

Ce à quoi le couple israélien franbréophone répond :

- Bonne année à vous et vos proches et espérons qu'un jour jije ktsat joter tov (ça ira un peu mieux)... L'espoir fait vivre c'est bien connu.
} 
Ou encore, deux amis, le franbréophone B et le francophone W. correspondent par mail :

Exemple 5.
W. pour information on a visité grâce à vous le magnifique, l'extraordinaire musée Louvre/Lens!!! Mazal tov! (bonne chance)
B. bravo les explorateurs Kol hakavod! (félicitations) (---) Chabat chalom ( bon chabat) et kol tuv (que tout se passe bien)! La prochaine fois visitez le musée 'la
Piscine' à Roubaix c'est aussi nekhmad (sympa)!

Ces quelques exemples montrent que le franbreu, de code exclusivement oral prend dans la réalité actuelle de la révolution communicationnelle une dimension écrite non négligeable. Cette dimension mérite un agenda d'études nouvelles qui s'attaqueraient à l'élaboration des aspects systématiques de ce franbreu écrit.

\section{Conclusion}

En conclusion, le français en Israël n'a jamais eu autant de locuteurs. Il évolue graduellement et à un rythme différent dans divers milieux vers ce code que nous avons appelé franbreu. Ce franbreu devient une réalité linguistique significative alors qu'arrivent des nouveaux venus se qualifiant de 'diaspora transnationale'. Pour ces immigrants l'insertion dans la société et l'acquisition de la langue dominante n'impliquent pas nécessairement l'abandon de la langue d'origine, même si celle-ci se transforme au contact de l'autre.

Ce modèle s'imprime dans la multiculturalisation de la société israélienne où participent également, pour ne citer qu'eux, l'hébreu, le russe, l'anglais ou l'arabe. Cette nouvelle configuration est marquée par la conjonction de traits sociologiques et sociolinguistiques qui forment un tableau d'ensemble assez cohérent d'une francophonie devenue, par le fait migratoire, une minorité.

\section{Bibliographie}

Ambassade de France (1995). Rapport de recherche. Tel-Aviv, Services culturels.

Ben-Rafael, E. / Ben-Rafael, M. (2013). Sociologie et Sociolinguistique des Francophonies Israéliennes. Frankfort, Berlin, NY : Peter Lang.

Ben-Rafael, E. ; Ben-Rafael, M. 2008. Linguistic landscape and transnationalism : Sarcelles-Natanya. Israel Studies in Language and Society, Electronic Interdisciplinary Journal 1 (1).

Ben-Rafael, E. / Sharot, S. (2007). Ethnicity, Religion and Class in Israel, Cambridge, New York, Cambridge University Press, Paperback re-print of the CUP hardcover (1991).

Ben-Rafael, E. / Sternberg, Y. (2009). La communauté franco-israélienne : composition, dispositions et structuration. Tel-Aviv : Les Services culturels, Ambassade de France.

Ben-Rafael, M. (1989). Interférence syntaxique et stratégies d'apprentissage: le français, langue étrangère en Israël, Université de Tel-Aviv : Département de français.

Ben-Rafael, M. (1994). «Interférences syntaxiques et stratégies d'apprentissage », in F.S. Martinez et al. (éds.), Las Lenguas en la Europa Communitaria. Amsterdam : Éditions Rodopi, 75-89.

Ben-Rafael, M. (2001a). Contact de langue : Le français parlé des francophones israéliens. Tel-Aviv: Université de Tel-Aviv.

Ben-Rafael, M. (2001b). Codeswitching in the language of immigrants: The case of Hebrew. In R. Jacobson (éd.), Codeswitching Worldwide II, New York : Mouton de Gruyter, 251-307. 
Ben-Rafael, M. / Schmid, M.S. (2007). Language attrition and ideology: Two groups of immigrants in Israel. In B. Köpke / M.S. Schmid / S. Keijzer / M. Dostert (éds.), Language Attrition : Theoretical Perspectives. Amsterdam : John Benjamins, 205-226.

Bentahila, A. (1983). Language attitudes among Arabic French Bilinguals in Morocco. Clevedon : Multilingual Matters.

Berk-Seligson, S. (1986). Linguistic constraints on intra-sequential code-switching: Study of Spanish/Hebrew bilingualism. In Language in Society, 15, 3113-348.

Boudras-Chapon, V. (2008). Langue nationale, langue officielle, langue vernaculaire, langue véhiculaire, langue maternelle. Riposte lä̈que, online: http://ripostelaique.com/Langue-nationale-langueofficielle.html

Fishman, J.A. / Kressel, R.H. (1974). The uses of Hebrew loan-words in spoken German in two bilingual communities. Linguistics 139, 69-78.

Garner-Chloros, P. (2009). Code-Switching, Cambridge, Cambridge University Press.

Glick Schiller, N. / Basch, L. / Blanc-Szanton, C. (1992). Towards a Transnational Perspective on Migration : Race, Class, Ethnicity, and Nationalism Reconsidered. New York : New York Academy of Sciences.

Grosjean, F. (1982). Life with two languages: An introduction to bilingualism, Cambridge : Harvard University Press.

Hofman, J.E. / Fisherman, H. (1972). Language shift and language maintenance in Israel. In J.A. Fishman (éd.), Advances in the Sociology of Language, The Hague : Mouton, vol. 2., 342-364.

Jacobson, R. (éd.). (1998-2001). Codeswitching Worldwide, vol. I et II. New York : Mouton de Gruyter.

Koplewitz, I. (1990). The use and integration of Hebrew lexemes in Israeli spoken Arabic. In D. Gorter / J.F. Hoekstra / L. Jansma / J. Ytsma (éds.), Fourth International Conference on Minority Languages, Clevedon : Multilingual Matters, vol. 2, 181-195.

Meyers-Scotton, C. (1991). Social motivations for code-switching. Oxford : Clarendon.

Myhill, J. 2006. Language, Religion and National Identity in Europe and the Middle East. Amsterdam / Philadelphia : PA, John Benjamins.

Olshtain, E. / Blum-Kulka, S. (1989). Happy Hebrish : Mixing and switching in American-Israeli Family interactions. In S. Gass / C. Madden / D. Preston / L. Selinker (éds.), Variation in second language acquisition discourse and pragmatics, Clevedon : Multinlingaul Matters, vol 1, 59-83.

Pergnier, M. (éd.) (1988). Le français en contact avec l'anglais. Paris : Didier Érudition.

Romaine, S. (1989). Bilingualism. Oxford : Blackwell.

Smooha, S. (1972). Israel : Pluralism and Conflict. London : Routledge and Kegan Paul.

Tabouret-Keller, A. (1985). Les corrélations sont muettes. La linguistique, 21,125-39.

Witte, E. / Beatens Beardsmore, H. (éds.) (1987). The Interdisciplinary Study of Urban Bilingualism in Brussels, Clevedon, Multilingual Matters.

\footnotetext{
${ }^{0}$ Nous pensons, par exemple, à la fréquentation des activités offertes par les services culturels de l'Ambassade de France à Tel-Aviv (séances de cinéma, bibliothèque, expositions etc.).

${ }^{1}$ Certains NVs sont nés en Afrique du nord et ont immigré en France avec leurs familles tandis que d'autres sont nés en France de parents d'origine nord-africaine. On note ici que près des deux tiers des Juifs résidant en France partagent ces mêmes origines.
} 


\begin{abstract}
${ }^{2}$ Depuis ces deux dernières décennies, le développement des médias, les facilités d'information et de communication offertes sur la toile, et les progrès de la télévision par câble et satellite ont accru pour beaucoup cette compétence en français standard et contemporain dans le domaine de la consommation culturelle.
\end{abstract}

